\title{
A FAMILY OF MAPS WITH MANY SMALL FIBERS
}

\author{
HANNAH ALPERT AND LARRY GUTH
}

\begin{abstract}
The waist inequality states that for a continuous map from $S^{n}$ to $\mathbb{R}^{q}$, not all fibers can have small $(n-q)$-dimensional volume. We construct maps for which most fibers have small $(n-q)$-dimensional volume and all fibers have bounded $(n-q)$-dimensional volume.
\end{abstract}

Let $n, q \in \mathbb{N}$ with $n>q \geq 1$, and let $f: S^{n} \rightarrow \mathbb{R}^{q}$ be a continuous map. Let $\widehat{p}: \mathbb{R}^{n+1} \rightarrow \mathbb{R}^{q}$ be a surjective linear map, and let $p=\left.\widehat{p}\right|_{S^{n}}$. The waist inequality states that the largest fiber of $f$ is at least as large as the largest fiber of $p$ :

$$
\sup _{y \in \mathbb{R}^{q}} \operatorname{Vol}_{n-q} f^{-1}(y) \geq \sup _{y \in \mathbb{R}^{q}} \operatorname{Vol}_{n-q} p^{-1}(y) .
$$

See [1], [3, 4], and [6] for proofs of the waist inequality, or [5] for a survey. In the case $q=1$, the waist inequality is a consequence of the isoperimetric inequality on $S^{n}$. The isoperimetric inequality can also be used to prove that the portion of $S^{n}$ covered by small fibers of $f$ is not very big; that is, for all $\varepsilon$, we have

$$
\operatorname{Vol}_{n} f^{-1}\left\{y: \operatorname{Vol}_{n-q} f^{-1}(y)<\varepsilon\right\} \leq \operatorname{Vol}_{n} p^{-1}\left\{y: \operatorname{Vol}_{n-q} p^{-1}(y)<\varepsilon\right\} .
$$

The theorem presented in this paper describes how the same statement does not hold in the case $q>1$. We have also included an appendix with a more precise statement of the waist inequality and the isoperimetric inequality.

Theorem 1. For every $n, q \in \mathbb{N}$ with $n>q>1$, and for every $\varepsilon>0$, there is a continuous map $f: S^{n} \rightarrow \mathbb{R}^{q}$ such that all but $\varepsilon$ of the $n$-dimensional volume of $S^{n}$ is covered by fibers that have $(n-q)$-dimensional volume at most $\varepsilon$. Moreover, we may require that every fiber of $f$ has $(n-q)$-dimensional volume bounded by $C_{n, q}$, a constant not depending on $\varepsilon$.

In what follows, $I^{n}=[0,1]^{n}$ denotes the $n$-dimensional unit cube, and $\partial I^{n}$ denotes its boundary. A tree refers to the topological space corresponding to a graphtheoretic tree: topologically, a tree is a finite 1-dimensional simplicial complex that is contractible.

The bulk of the construction comes from the following lemma, in which we construct a preliminary "tree map" $t_{n, r, \delta}$ from $I^{n}$ to a tree. Later, to construct $f$ we will change the domain from $I^{n}$ to $S^{n}$ by gluing several tree maps together, and we will change the range from the tree to $\mathbb{R}^{q}$ by composing with a map from a thickened tree to $\mathbb{R}^{q}$. In the tree map $t_{n, r, \delta}$, the parameter $r$ corresponds to the depth of the tree. As $r$ increases, the typical fiber of the map becomes smaller. The parameter $\delta$ corresponds to the total volume of the larger fibers.

Lemma 1. For every $n, r \in \mathbb{N}$, there is a rooted tree $T_{n, r}$ such that for every $\delta>0$ there is a continuous map $t_{n, r, \delta}: I^{n} \rightarrow T_{n, r}$ with the following properties:

2010 Mathematics Subject Classification. 53C23. 


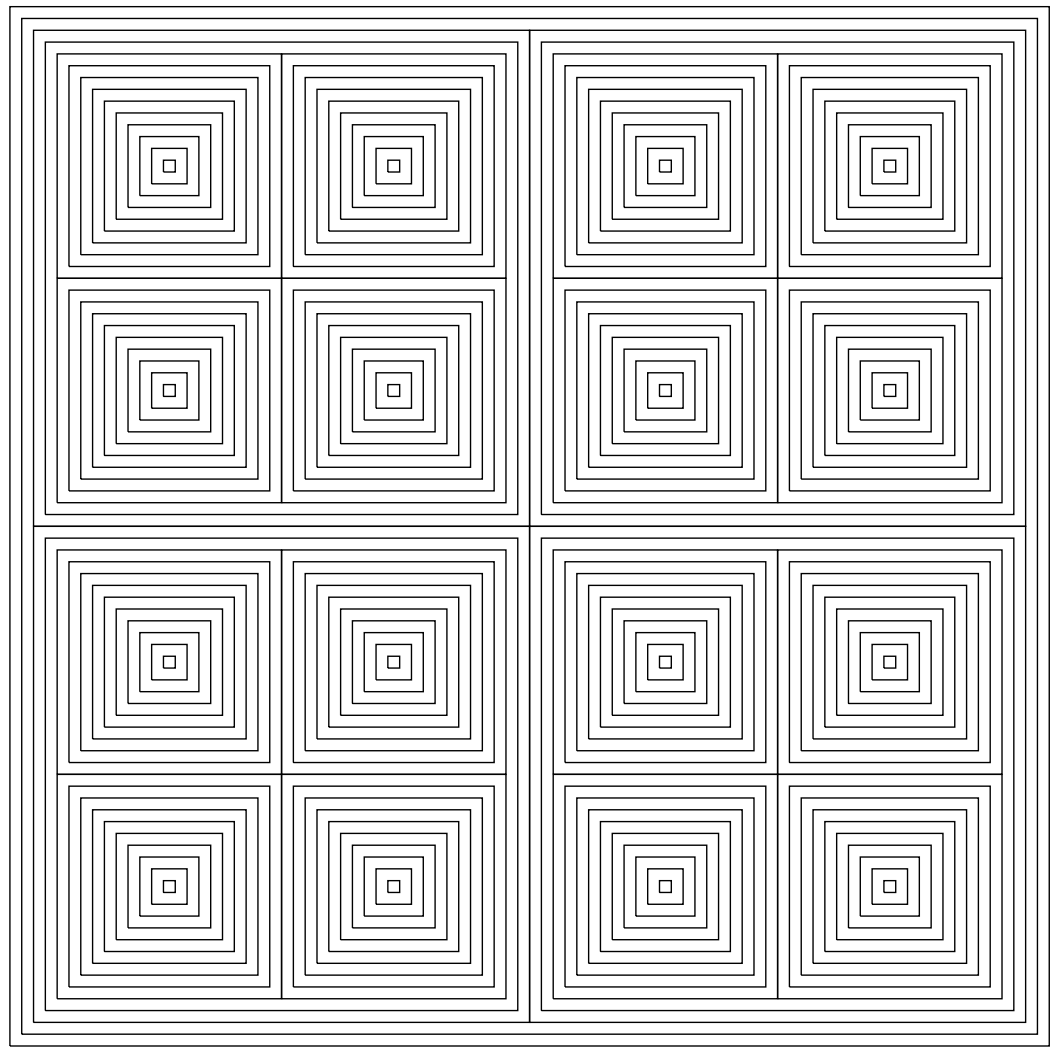

FIGURE 1. Every fiber of $t_{2,2, \delta}$ has length at most 6 , and most fibers have length at most 1.

(1) Every fiber of $t_{n, r, \delta}$ is either a single point, the boundary of an $n$-dimensional cube of side length at most 1 , or the $(n-1)$-skeleton of a $2 \times 2 \times \cdots \times 2$ array of $n$-dimensional cubes each of side length at most $\frac{1}{2}$.

(2) All but $\delta$ of the volume of $I^{n}$ is covered by fibers of $t_{n, r, \delta}$ that are boundaries of $n$-dimensional cubes of side length at most $2^{-r}$.

(3) $t_{n, r, \delta}\left(\partial I^{n}\right)$ is a single point, the root of $T_{n, r}$.

(4) Each vertex has at most $2^{n}$ daughter vertices.

Proof. We construct the tree and tree map recursively in $r$. For $r=0$, the tree $T_{n, 0}$ is a single edge which we may identify with the interval $\left[0, \frac{1}{2}\right]$, with 0 being the root. For any $\delta$, we set $t_{n, 0, \delta}(x)=\operatorname{dist}\left(x, \partial I^{n}\right)$ for all $x \in I^{n}$.

Now let $r>0$. To construct $T_{n, r}$, we take the disjoint union of one copy of $[0,1]$ and $2^{n}$ copies of $T_{n, r-1}$, and identify the root of every copy of $T_{n, r-1}$ with $1 \in[0,1]$. The root of $T_{n, r}$ is $0 \in[0,1]$. We define $t_{n, r, \delta}$ piecewise as follows. For some small choice of $\delta_{1}>0$, we define $t_{n, r, \delta}$ on the closed $\delta_{1}$-neighborhood of $\partial I^{n}$ to $[0,1] \subset T_{n, r}$ by

$$
t_{n, r, \delta}(x)=\frac{1}{\delta_{1}} \operatorname{dist}\left(x, \partial I^{n}\right) .
$$


Then, translating the coordinate hyperplanes to pass through the center of $I^{n}$ we divide the remainder of the cube into a $2 \times 2 \times \cdots \times 2$ array of cubes $Q_{1}, \ldots, Q_{2^{n}}$ each of side length slightly less than $\frac{1}{2}$. For each $j=1, \ldots 2^{n}$, let $\lambda_{j}: Q_{j} \rightarrow I^{n}$ be the map that scales $Q_{j}$ up to unit size, and let $i_{j}: T_{n, r-1} \rightarrow T_{n, r}$ be the inclusion of the $j$ th copy of $T_{n, r-1}$ into $T_{n, r}$. Then for some small choice of $\delta_{2}>0$, we put

$$
\left.t_{n, r, \delta}\right|_{Q_{j}}=i_{j} \circ t_{n, r-1, \delta_{2}} \circ \lambda_{j} .
$$

Properties 1,3 , and 4 are easily satisfied by the construction. To ensure property 2 , we need to choose $\delta_{1}$ and $\delta_{2}$. The volume of $I^{n}$ that is covered by large fibersfibers not equal to the boundary of a cube of side length at most $2^{-r}$-is at most $\delta_{1} \cdot 2 n+2^{n} \cdot \delta_{2} \cdot 2^{-n}$, because the area of $\partial I^{n}$ is $2 n$ and because the portion of each $Q_{j}$ that is covered by large fibers has volume at most $\delta_{2} \cdot \operatorname{Vol}\left(Q_{j}\right)<\delta_{2} \cdot 2^{-n}$. Thus we may choose $\delta_{1}=\frac{\delta}{4 n}$ and $\delta_{2}=\frac{\delta}{2}$.

Proof of Theorem 1. We may replace $S^{n}$ by $\partial I^{n+1}$ by composing with the (biLipschitz) homeomorphism $\psi: S^{n} \rightarrow \partial I^{n+1}$ given by lining up the centers of $S^{n}$ and $\partial I^{n+1}$ in $\mathbb{R}^{n+1}$ and projecting radially. We start by constructing a tree $T$ and a tree map $t: \partial I^{n+1} \rightarrow T$. For some large choice of $r$, let $T$ be the tree obtained by identifying the roots of $2(n+1)$ copies of $T_{n, r}$, one for each $n$-dimensional face of $\partial I^{n+1}$. For some small choice of $\delta$, define $t$ on each $n$-dimensional face of $\partial I^{n+1}$ to be the composition of $t_{n, r, \delta}$ with the inclusion of the corresponding $T_{n, r}$ into $T$.

The fibers of $t$ have dimension $n-1$. In order to cut the fibers down to dimension $n-q$, we next construct a projection map $p: \partial I^{n+1} \rightarrow \mathbb{R}^{q-1}$ such that the fibers of $p$ intersect the fibers of $t$ transversely. The fibers of $t$ have codimension 2 in $\mathbb{R}^{n+1}$ and are aligned with the standard coordinates, so we achieve transversality by using other linear coordinates to construct $p$. We choose $q-1$ linearly independent vectors $v_{1}, \ldots, v_{q-1} \in \mathbb{R}^{n+1}$ such that for every two standard basis vectors $e_{i}, e_{j} \in \mathbb{R}^{n+1}$ the spaces $\operatorname{span}\left\{e_{i}, e_{j}\right\}^{\perp}$ and $\operatorname{span}\left\{v_{1}, \ldots, v_{q-1}\right\}^{\perp}$ intersect transversely; equivalently, the set $e_{i}, e_{j}, v_{1}, \ldots, v_{q-1}$ is linearly independent. For $k=1, \ldots, q-1$, define the $k$ th component of $p$ to be the dot product of the input with $v_{k}$. Then the fibers of $t \times p: \partial I^{n+1} \rightarrow T \times \mathbb{R}^{q-1}$ are codimension $q-1$ transverse linear cross-sections of the $(n-1)$-dimensional fibers of $t$, and have $(n-q)$-dimensional volume bounded by some constant depending on $n$ and $q$.

There exists $M$ large enough that $p\left(\partial I^{n+1}\right)$ is contained in the $(q-1)$-dimensional ball $B(M)$ of radius $M$. We define a map $\phi: T \times B(M) \rightarrow \mathbb{R}^{q}$ such that the number of points in each fiber of $\phi$ is at most the maximum degree of $T$, which is $2^{n}+1$. Then we define $f=\phi \circ(t \times p)$. The fibers of $f$, like the fibers of $t \times p$, have $(n-q)$-dimensional volume bounded by a constant $C_{n, q}$.

The map $\phi$ is constructed as follows. Let $\left.\phi\right|_{T \times\{0\}}$ be an embedding of $T$ into $\mathbb{R}^{q}$ in which the edges map to straight line segments and each daughter vertex has $x_{1}$ coordinate greater than that of its parent. Let $d$ be the minimum distance between disjoint edges of $\phi(T \times\{0\})$. Then for every $p \in T$ and $x \in B(M)$, we set

$$
\phi(p, x)=\phi(p, 0)+\frac{d}{4}\left(0, \frac{x}{M}\right)
$$

where $\left(0, \frac{x}{M}\right)$ denotes the point in $\mathbb{R}^{q}$ constructed by adding onto $\frac{x}{M} \in \mathbb{R}^{q-1}$ a first coordinate of 0 . If $\phi(p, x)=\phi\left(p^{\prime}, x^{\prime}\right)$, then $\phi(p, 0)$ and $\phi\left(p^{\prime}, 0\right)$ are at most $\frac{d}{2}$ apart, so $p$ and $p^{\prime}$ lie on two incident edges of $T$; also, $\phi(p, 0)$ and $\phi\left(p^{\prime}, 0\right)$ have the same 
$x_{1}$-coordinate, so these two edges are between two daughters and a common parent, rather than a daughter, a parent, and a grandparent.

To finish the proof, we show that $\delta$ and $r$ may be chosen such that all but $\varepsilon$ of the $n$-dimensional volume of $\partial I^{n+1}$ is covered by fibers with $(n-q)$-dimensional volume at most $\varepsilon$. The maximum number of daughter vertices of any vertex of $T$ is $2^{n}$, and most of $\partial I^{n+1}$ is covered by fibers of $f$ that are unions of at most $2^{n}$ codimension $q-1$ transverse linear cross-sections of boundaries of $n$-dimensional cubes of side length at most $2^{-r}$. We choose $r$ large enough that every codimension $q-1$ transverse linear cross-section of $2^{-r} \partial I^{n}$ has $(n-q)$-dimensional volume at most $\frac{\varepsilon}{2^{n}}$. The volume of the portion of $\partial I^{n+1}$ covered by larger fibers is at most $2(n+1) \cdot \delta$, so we choose $\delta<\frac{\varepsilon}{2(n+1)}$.

\section{APPENDIX: THE WAIST INEQUALITY AND THE ISOPERIMETRIC INEQUALITY}

In order to be precise about the waist inequality, we need a notion of $(n-q)$ dimensional volume of arbitrary closed subsets in $S^{n}$. Gromov's version of the waist inequality is stated in terms of the Lebesgue measures $\mathrm{Vol}_{n}$ of the $\varepsilon$-neighborhoods $f^{-1}(y)_{\varepsilon}$ of the fibers $f^{-1}(y)$ of a continuous map $f$.

Theorem 2 (Waist inequality, [4]). Let $f: S^{n} \rightarrow \mathbb{R}^{q}$ be a continuous map. Then there exists a point $y \in \mathbb{R}^{q}$ such that for all $\varepsilon>0$, we have

$$
\operatorname{Vol}_{n}\left(f^{-1}(y)_{\varepsilon}\right) \geq \operatorname{Vol}_{n}\left(S_{\varepsilon}^{n-q}\right),
$$

where $S^{n-q} \subset S^{n}$ denotes an equatorial $(n-q)$-sphere.

The paper [6] gives a detailed exposition of the proof of the waist inequality and fills in some small gaps in the original argument. For convenience we introduce a notation for comparing the $\varepsilon$-neighborhoods of two sets: given $E, F \subseteq S^{n}$, we say that $E$ is larger in neighborhood than $F$, denoted $E \geq_{\text {nbd }} F$, if for all $\varepsilon>0$ we have

$$
\operatorname{Vol}_{n}\left(E_{\varepsilon}\right) \geq \operatorname{Vol}_{n}\left(F_{\varepsilon}\right)
$$

Then the waist inequality states that for some $y \in \mathbb{R}^{q}$ we have $f^{-1}(y) \geq_{\text {nbd }} S^{n-q}$.

In the case $q=1$, we would like to say that the waist inequality is a consequence of the isoperimetric inequality. The classical isoperimetric inequality applies only to regions with smooth boundary, so we need the following version, which is stated and proved in [2] and attributed to [7:

Theorem 3 (Isoperimetric inequality). Let $A \subseteq S^{n}$ be a closed set and $B \subseteq S^{n}$ be a closed ball with $\operatorname{Vol}_{n}(B)=\operatorname{Vol}_{n}(A)$. Then we have

$$
A \geq \text { nbd } B \text {. }
$$

In the introduction we claimed that in the case $q=1$, the isoperimetric inequality could be used to prove, in addition to the waist inequality, another statement about the volume of $S^{n}$ covered by small fibers. Here we formulate the statement more precisely and prove it. The proof implies the waist inequality for $q=1$.

Theorem 4. Let $f: S^{n} \rightarrow \mathbb{R}$ be a continuous map, and $p: S^{n} \rightarrow \mathbb{R}$ be the restriction to $S^{n}$ of a surjective linear map $\widehat{p}: \mathbb{R}^{n+1} \rightarrow \mathbb{R}$. Then for all $y \in p\left(S^{n}\right)$, we have

$\operatorname{Vol}_{n}\left\{x \in S^{n}: f^{-1}(f(x)) \geq_{\text {nbd }} p^{-1}(y)\right\} \geq \operatorname{Vol}_{n}\left\{x \in S^{n}: p^{-1}(p(x)) \geq_{\text {nbd }} p^{-1}(y)\right\}$.

The proof of this theorem is based on the following lemma: 
Lemma 2. Let $X, Y \subset S^{n}$ be closed sets with $X \cup Y=S^{n}$. Let $B^{X}, B^{Y} \subset S^{n}$ be closed balls such that their two centers are antipodal in $S^{n}$ and $\operatorname{Vol}_{n}\left(B^{X}\right)=\operatorname{Vol}_{n}(X)$ and $\operatorname{Vol}_{n}\left(B^{Y}\right)=\operatorname{Vol}_{n}(Y)$. Then we have

$$
X \cap Y \geq_{\mathrm{nbd}} B^{X} \cap B^{Y} .
$$

Proof. First we claim that $(X \cap Y)_{\varepsilon}$ is the disjoint union of $X_{\varepsilon} \backslash X, Y_{\varepsilon} \backslash Y$, and $X \cap Y$. It is clear that $(X \cap Y)_{\varepsilon}$ is the disjoint union of its intersections with $S^{n} \backslash X$, $S^{n} \backslash Y$, and $X \cap Y$. Thus it suffices to show that

$$
(X \cap Y)_{\varepsilon} \cap\left(S^{n} \backslash X\right)=X_{\varepsilon} \backslash X .
$$

Because $(X \cap Y)_{\varepsilon} \subseteq X_{\varepsilon}$, we immediately have

$$
(X \cap Y)_{\varepsilon} \cap\left(S^{n} \backslash X\right) \subseteq X_{\varepsilon} \backslash X .
$$

For the reverse inclusion, let $y \in X_{\varepsilon} \backslash X$, and let $\gamma:[0,1] \rightarrow S^{n}$ be a curve of length at most $\varepsilon$ with $\gamma(0)=y$ and $\gamma(1)=x \in X$. Let $t \in[0,1]$ be the greatest value with $\gamma(t) \in Y$. Then $\gamma(t) \in X \cap Y$, so $y \in(X \cap Y)_{\varepsilon}$.

Thus, applying the isoperimetric inequality and additivity of measure, we have

$$
\begin{aligned}
& \operatorname{Vol}_{n}\left((X \cap Y)_{\varepsilon}\right)=\operatorname{Vol}_{n}\left(X_{\varepsilon}\right)-\operatorname{Vol}_{n}(X)+\operatorname{Vol}_{n}\left(Y_{\varepsilon}\right)-\operatorname{Vol}_{n}(Y)+\operatorname{Vol}_{n}(X \cap Y) \geq \\
& \quad \geq \operatorname{Vol}_{n}\left(B_{\varepsilon}^{X}\right)-\operatorname{Vol}_{n}\left(B^{X}\right)+\operatorname{Vol}_{n}\left(B_{\varepsilon}^{Y}\right)-\operatorname{Vol}_{n}\left(B^{Y}\right)+\operatorname{Vol}_{n}\left(B^{X} \cap B^{Y}\right)= \\
& \quad=\operatorname{Vol}_{n}\left(\left(B^{X} \cap B^{Y}\right)_{\varepsilon}\right) .
\end{aligned}
$$

Proof of Theorem 4. Without loss of generality we assume $p\left(S^{n}\right)=[0,1]$ and $y \leq \frac{1}{2}$. Then on the right-hand side of the desired inequality we have

$$
\left\{x \in S^{n}: p^{-1}(p(x)) \geq_{\text {nbd }} p^{-1}(y)\right\}=p^{-1}[y, 1-y] .
$$

Define $\alpha, \beta \in \mathbb{R}$ as

$$
\begin{gathered}
\alpha=\sup \left\{t \in \mathbb{R}: \operatorname{Vol}_{n} f^{-1}(-\infty, t) \leq \operatorname{Vol}_{n} p^{-1}[0, y]\right\}, \\
\beta=\inf \left\{t \in \mathbb{R}: \operatorname{Vol}_{n} f^{-1}(t, \infty) \leq \operatorname{Vol}_{n} p^{-1}[y, 1]\right\} .
\end{gathered}
$$

For each $t \in[\alpha, \beta]$, apply the lemma with $X=f^{-1}(-\infty, t]$ and $Y=f^{-1}[t, \infty)$ to get $f^{-1}(t) \geq_{\text {nbd }} p^{-1}\left[y_{1}, y_{2}\right]$ for some $y_{1}, y_{2} \in[y, 1-y]$. In particular, we have

$$
f^{-1}(t) \geq_{\text {nbd }} p^{-1}\left(y_{1}\right) \geq_{\text {nbd }} p^{-1}(y) .
$$

Thus, we have

$$
f^{-1}[\alpha, \beta] \subseteq\left\{x \in S^{n}: f^{-1}(f(x)) \geq_{\text {nbd }} p^{-1}(y)\right\} .
$$

Because $\operatorname{Vol}_{n} f^{-1}(-\infty, \alpha) \leq \operatorname{Vol}_{n} p^{-1}[0, y]$ and $\operatorname{Vol}_{n} f^{-1}(\beta, \infty) \leq \operatorname{Vol}_{n} p^{-1}[y, 1]$ we have

$$
\operatorname{Vol}_{n} f^{-1}[\alpha, \beta] \geq \operatorname{Vol}_{n} p^{-1}[y, 1-y]
$$




\section{REFERENCES}

[1] F.J. Almgren, The theory of varifolds - a variational calculus in the large for the $k$ dimensional area integrated, Mimeographed notes, 1965.

[2] T. Figiel, J. Lindenstrauss, and V. D. Milman, The dimension of almost spherical sections of convex bodies, Acta Math. 139 (1977), no. 1-2, 53-94. MR 0445274 (56 \#3618)

[3] M. Gromov, Filling Riemannian manifolds, J. Differential Geom. 18 (1983), no. 1, 1-147. MR 697984 (85h:53029)

[4] - Isoperimetry of waists and concentration of maps, Geom. Funct. Anal. 13 (2003), no. 1, 178-215. MR 1978494 (2004m:53073)

[5] L. Guth, The waist inequality in Gromov's work, The Abel Prize 2008-2012 (H. Holden and R. Piene, eds.), Springer, 2014, pp. 181-195.

[6] Y. Memarian, On Gromov's waist of the sphere theorem, J. Topol. Anal. 3 (2011), no. 1, 7-36. MR 2784762 (2012g:53066)

[7] E. Schmidt, Die Brunn-Minkowskische Ungleichung und ihr Spiegelbild sowie die isoperimetrische Eigenschaft der Kugel in der euklidischen und nichteuklidischen Geometrie. I, Math. Nachr. 1 (1948), 81-157. MR 0028600 (10,471d)

MiT, Cambridge, MA 02139 USA

E-mail address: hcalpert@math.mit.edu

E-mail address: lguth@math.mit.edu 